Final Report

submitted to the

National Aeronautics and SpaCe Administration Shuttle Contract Office, Processing and Consumables Branch OP-SCO-1

Kennedy Space Center 32899

ATTN: JoyCE E. BeEson, Grants Specialist

entitled

\title{
Development and verification of methods for predicting flow rates through leaks in valves and couplings
}

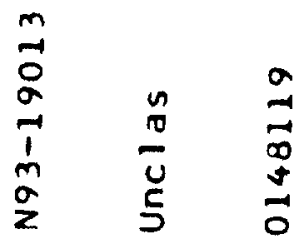 \\ prepared by \\ JOHN M. RUSSELL \\ Associate Professor \\ Department of Mechanical and Aerospace Engineering \\ submitted by \\ Florida InStitute of TEChNOLOGY \\ Office of Sponsored Programs \\ 150 West University Boulevard \\ Melbourne, Florida, 32901 \\ (407) 768-8000, x7269 \\ Grant number: NAG10-0089 \\ Grant period: September 15, 1991 to January 31, 1993
}

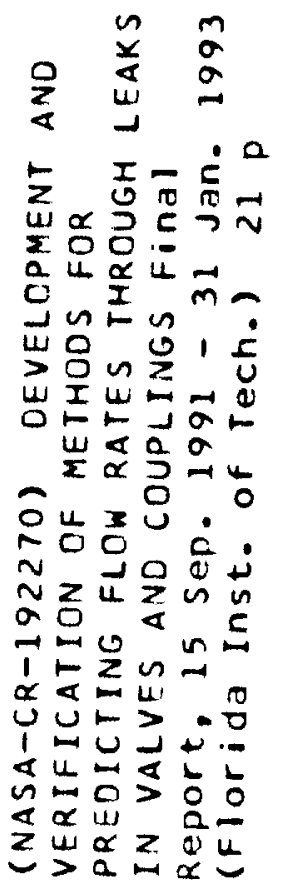

March 8, 1993 


\title{
Development and verification of methods for predicting flow rates through leaks in valves and couplings
}

\author{
By
}

JOHN M. RUSSELL

Florida Institute of Technology

\begin{abstract}
This is the final report of a research effort which addresses the title problem. The report discusses two broad models of flows, which represent the extreme cases of: (i) inertia-dominated flow, where friction is relatively insiginificant; and (ii) friction-dominated flow where inertia is insignificant. In class (ii), the leak channel might consist of a the gap between a scratch in a plastic seal and a polished metal plate against which the seal is pressed. Here, the cross section of the leak channel is modeled as a flat bottomed crescent. A publication generated under the present grant period presents an exact solution of the equations of fullydeveloped laminar pipe flow of a liquid in the case of a crescent beneath a hyperbolic arc. A Master's thesis project supported by the present grant presents the corresponding solution beneath a circular arc. A second publication reviews the flow of a gas through the same channel, which may be analyzed by a standard one-dimensional model (FANNo flow) for an engineering approximation. Finally, the report discusses the design and progress in the fabrication of a leak-test cell, in which one may measure the flow of fluid through a controlled flaw in a seal. The aim of such measurements is to furnish data for comparison with the predictions of the theory.
\end{abstract}

Key words: laminar flow, pipe flow, leakage, cryogenic fluids 


\section{SUMMARY}

Valves, couplings, and other devices meant to control the flow of liquid hydrogen are subject to quality-control testing before they are put into service at Kennedy Space Center. The hazards posed by the flammability of liquid hydrogen and the costs of overcoming them have led to the development of operational criteria for leak tightness based on the rate of leakage of an inert gas (such as helium) at a representative cryogenic temperature (such as that of liquid nitrogen). The present work arose from an attempt to derive a correlation between the measured flow rate of such an inert working fluid and the corresponding flow rate of liquid hydrogen through a leak of similar geometry and pressure difference between the inlet and the outlet.

A typical cryogenic seal is made of a plastic such as polytetrafluroethelene (PTFE) and is clamped between polished metal parts. If there is a scratch on the plastic sealing surface prior to installation, then, the region between the seal and the plate against which it is pressed during installation may form a leak channel. The cross section of such a channel is noncircular, and one is led to model it by a simple shape such as a flat bottomed crescent (i.e. the region between a circular or hyperbolic arc and a line drawn through its endpoints).

The equations of fully developed laminar flow of a liquid through a pipe of arbitrary cross section are well known. So also is the solution in the case of a few simple boundary shapes, such as the ellipse, the rectangle, and the equilateral triangle (to name three examples). When the shape of the cross section is a flat bottomed crescent, one must solve the equations anew and solutions in the case of circular and hyperbolic crescents have been found during the work reported herein. A full derivation of the solutions in the case of a hyperbolic cresecent is furnished in a separate publication (AIAA Paper 93-0559, January 1993) produced during the present grant period. The corresponding solution in the case of a circular crescent is presented in the Master's thesis of WENDY S. SMITH (Department of Mechanical and Aerospace Engineering, FIT, Winter term 1993). This latter work was supported by the present grant in the form of a one-year research assistanthip.

When the fluid is a gas, the exact equations of motion, owing to their mathematical complexity, are not amenable to analytic solution. A one-dimensional model based on cross stream averaging of the equations and the flow variables (FANNo flow) leads to an engineering approximation of the flow which suffices for many purposes. A careful rederivation of this model is presented in a paper produced during the present grant peroid. This paper was presented at the First World Congress of Nonlinear Analysts in Tampa, August 19-26, 1993 and a written version of it has been accepted for publication in the Proceedings of that Congress (to be published by the Walter de Gruyter Company).

The foregoing flow models correspond to the idealization of pipe flow, in which frictional effects balance pressure gradients and inertia plays a secondary role. Such a model makes sense if the leak channel is in the form of a slender scratch whose cross sectional shape and size do not vary appreciably over a distance of several scratch widths. Another model is one in which the basic balance is between inertia and pressure gradients and frictional effects are secondary. This latter model makes sense if the leak channel has the form of a contraction leading to a throat whose length is not long compared to its width. The frictionless model is far simpler to formulate than the frictional one, so the discussion of the frictional case is preceeded by a discussion of the frictionless case in this report.

In both of the flow models discussed above, pathologies such as adiabatic boiling, heat transfer, and inlet-swirl effects are ignored. It is not clear how important such effects are in practice, but their presence may explain discrepancies between predictions of theoretical models and the results of experimental measurements.

The next major part of this report discusses the design and progress toward the fabrication of a leak-test cell for measuring the rate of flow of a cryogenic fluid through a controlled flaw in a seal. When expressed in terms of full-time equivalent man weeks over the whole grant period, the Principal Investigator was supported for a total of nineteen and three-quarters man-weeks of effort. About nine of these man-weeks were spent on the engineering design of a leak-test cell and the procurement of its major components. The device consists of a 
flat stainles steel plate (AISI type 304) to which two stainless steel cups are bolted, one inside of the other. Fluid lines are to be run through the plate and connected to laboratory supplies of, say, liquid nitrogen and gaseous helium and tests are to be conducted while the whole apparatus is submerged in a bath of liquid nitrogen. The gasket between the inner cup and the plate is to have a controlled flaw in it. Such a flaw may be produced by placing a fine wire across the gasket prior to installation and leaving it there during the subsequent tests. The gap between the inner and the outer cups captures the leaked fluid and allows it to be measured either by direct mechanical means (e.g., a small volume-flow-rate meter) or by chemical means (e.g., a mass spectrometer).

The rates of thermal contraction of gasket material and steel are, of course, unequal, which raises the question of how to maintain bolt tension between the time the bolts are first tightened (at room temperature) and the time the tests are conducted (at cryogenic temperature). The solution chosen in the present design is to adjoin a stack of BELLVILLE springs (also known as disk springs) to the stack of objects to be fastened by the bolts. With judicious use of BELLEviLLE springs, one can arrange that ninety percent of the original room-temperature bolt tension is maintained after cryogenic cool down.

The two cups and the plate have been fabricated (by a tool and dye shop) at a cost of $\$ 1,025.00$ to the grant. The BELLVILLE springs, gaskets, bolts, and nuts have all been procured. Work yet to be done includes the installation of fittings to run fluid lines through the plate and the troubleshooting of the apparatus as a whole (in particular the verification that the installation of fluid-line fittings does not introuduce new leak paths). The intent of the principal investigator is, of course, to carry out this work at KSC at some future date with the advice and assistance of KSC personnel using facilities in place there (particularly the small cold box under the control of Mr. JAMES FESMIRE) and to test the forcasts of the theory with measurements obtained with the new leak-test cell. 


\section{CONTENTS}

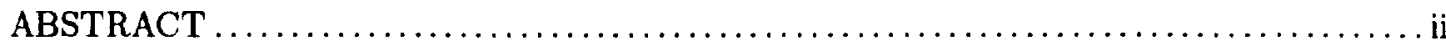

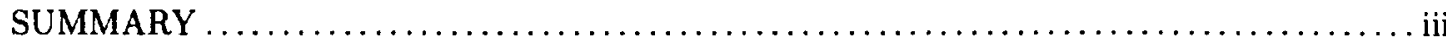

1.

1.1

1.2

1.3

1.4

1.5

2.

INTRODUCTION

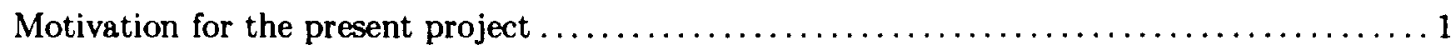

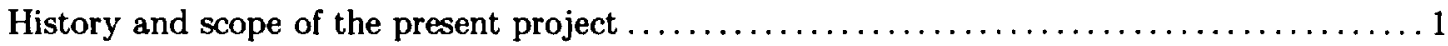

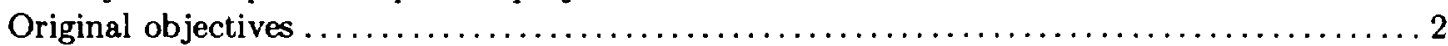

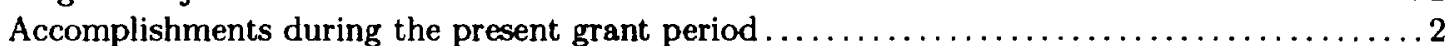

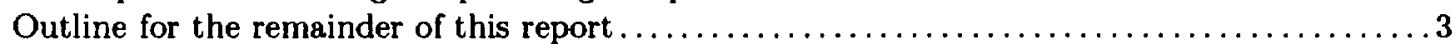

MODELS AND SOME PREDICTIONS MADE BY THEM $\ldots \ldots \ldots \ldots \ldots \ldots \ldots ., \ldots$

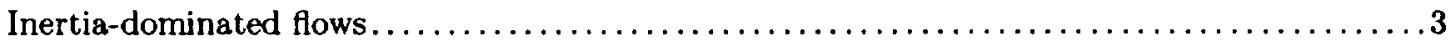

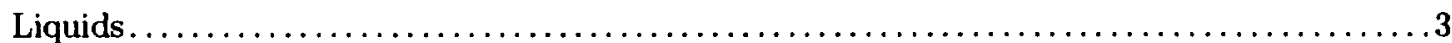

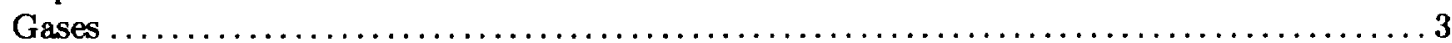

Liquid to gas mass flow rate ratio versus reservoir pressure $\ldots \ldots \ldots \ldots \ldots \ldots \ldots \ldots \ldots \ldots$

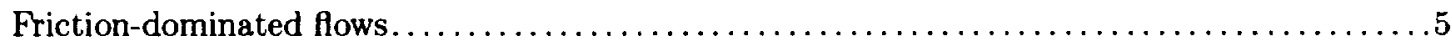

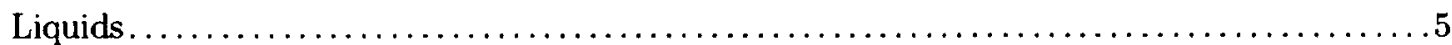

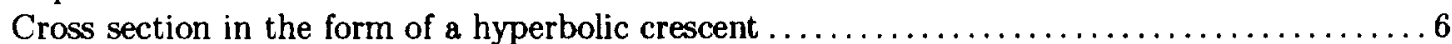

Formulas and tables for application of the hyperbolic-crescent model $\ldots \ldots \ldots \ldots \ldots \ldots \ldots .7$

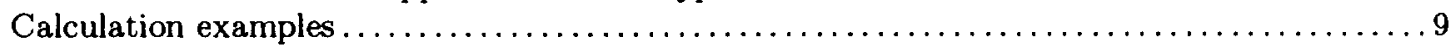

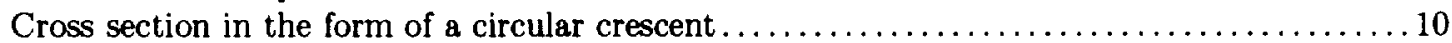

Gases ........................................................... 11

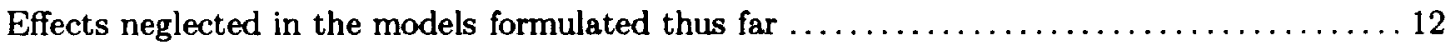

DESIGN AND FABRICATION OF A LEAK-TEST CELL $\ldots \ldots \ldots \ldots \ldots \ldots \ldots 13$

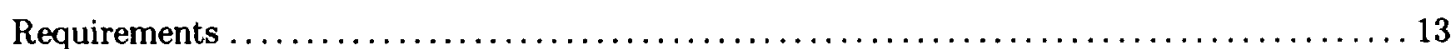

Factors leading to the choice of the present configuration...........................

RECOMMENDATIONS FOR FOLLOW-ON WORK $\ldots \ldots \ldots \ldots \ldots \ldots \ldots \ldots \ldots \ldots$

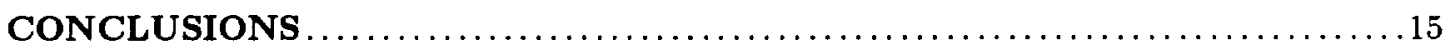

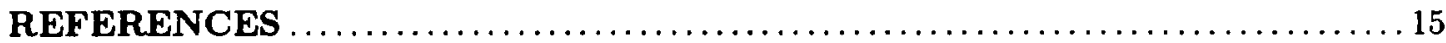




\section{INTRODUCTION}

\subsection{MOTIVATION FOR THE PRESENT PROJECT}

Valves, couplings, and other devices meant to control the flow of liquid hydrogen are subject to qualitycontrol testing before they are put into service at Kennedy Space Center. Hydrogen is, of course, flammable when mixed with air. The flammability of hydrogen-air mixtures raises the hazard of fires and explosions in all qualitity-control testing of components that are designed for use with hydrogen as the working fluid. In cases where the purpose of a test is merely to assure leak tightness of a given component within broad criteria, the realism gained by using hydrogen as the working fluid may not be worth the cost of overcoming the hazards of fire and explosions.

Such considerations have led to the development of operational criteria for leak tightness based on the rate of leakage of an inert gas (such as helium) at a representative cryogenic temperature (such as that of liquid nitrogen). A question that arises naturally under this circumstance is:

Given an inlet pressure, leak channel geometry, and receiver pressure, is there a way to correlate the mass flow rate of gaseous helium under these conditions with that of liquid hydrogen?

The present project was motivated by this question.

There is some NASA literature from the Apollo era on the problem. Thus, in chapter 2 (titled "Leakage") of a NASA-SP publication from 1967 (Reference 4, titled NASA contributions to advanced valve technology), J.W. MarR (physical chemist, Research and Development Center, General Electric Company, Schenectady) addresses a question similar to the one posed above. Although he obtains some interesting preliminary results, his model of compressible pipe flow seem to be too crude to be useful in cases where there is sonic choking. Thus, MARR assumes that the incompressible form of the PoISEIULLE pipe-flow formulas may be applied in the case of gas flow provided one replaces the mass density (constant in incompressible flow) by an effective value calculated (under an isothermal assumption) by averaging the inlet and outlet pressures. This model is cruder than the more familiar one-dimensional one (associated with the name FaNNO) described in standard reference works (e.g., the two-volume work of SHAPIRo, Reference 5). One may surmise that there is a continuing need for further work that addresses the above italicized question.

\subsection{HISTORY AND SCOPE OF THE PRESENT PROJECT}

The principal investigator of the present project (JMR) spent Summer 1989 at the Kennedy Space Center supported by a NASA/ASEE Faculty Summer Research Fellowship. His NASA colleague at the time was W. IRBY MOORE, who was in charge of the Propellents and Gases Branch (DM-MED-4). At the end of Summer $1989, \mathrm{Mr}$. MoORE suggested to JMR that a study of the correlation between the leak flow rate of gaseous helium and the leak flow rate of liquid hydrogen through a leak path of the same geometry and similar end-state pressures would be useful. This suggestion led to the submission of a proposal titled "The development and verification of methods for predicting flow rates through leaks in valves and couplings" dated September 15, 1989. This proposal received favorable evaluation on technical grounds, but budgetary restraints prevented funding at that time. On March 22,1991, JMR was apprised of a change in the funding situation and invited to submit an updated version of the same proposal. He therefore submitted a second version on April 3,1991. At the sponsor's request, a third version (one with a revised budget) was submitted on April 24, 1991, and that version was the one that resulted in the award of Grant NAG10-0089 for $\$ 65,000$.

The original grant period was September 15, 1991 to September 15, 1992. Upon JMR's request, a no-cost extension was granted which extended the closing date to January $31,1993$.

The budget of the present project provided suport for the principal investigator (JMR) at a quarter-time rate for 39 weeks (one academic year at FIT) and a full-time rate for ten summer weeks during 1992. This allocation is equivalent to nineteen and three-quarters man-weeks at a full-time rate. The budget also allocated a stipend for a graduate research assistant at flat rate of $\$ 1,828.44$ for each of four quarters. If one assumes a compensation rate of $\$ 15.00$ per hour, the reimbursed support for the graduate assistant amounted 
to 488 hours, or the equivalent of 12.2 man-weeks of full-time effort. Other charges to the grant included tuition remission for the graduate assistant, travel, purchase of a Macintosh IIci computer with related peripherals and software, overhead charges by FIT, and other charges itemized in the original proposal. A line item from the original budget, titled "Data processing charges" $(\$ 1,000$.) was never needed for that purpose. Instead, an amendment to the budget, moving those funds from "Data processing" to "Capital equipment", was proposed to NASA and approved in September 1992. This modified Capital Equipment budget paid for the fabrication of the major components of the leak-test cell built during the present grant period.

\subsection{ORIGINAL OBJECTIVES}

According to the original proposal for this project (which was submitted September 15, 1989 and resubmitted in revised form April 24, 1991) the objectives were:

$i$. To prepare a critical exposition of analytical methods by means of which engineers may predict the rate of flow of a given fluid (possibly cryogenic) through a given orifice that models a leak in a valve or coupling and which is subject to a given overall pressure drop from the inside to the outside of the pipe;

ii. To modify or extend those methods to allow for deformation of compliant boundaries such as seals or seal assemblies; and

iii. To establish the range of validity of the methods thus identified by comparing their predictions with suitable test data. Such data, if not already in print, will be generated by experimentation during this project.

\subsection{ACCOMPLISHMENTS DURING THE PRESENT GRANT PERIOD}

Objective $i$ has been effectively achieved and the "critical exposition" mentioned in it is to be found in two papers by RUSSELL (References $1 \& 2$ ) and in Master's thesis by SMITH (Reference 3). Objective $i i$, to include the direct effects of compliant boundaries in flow-rate estimation techniques, has been given relatively little attention in this project, since the principal investigator no longer believes that the inclusion of such effects would lead to a gain in prediction accuracy commensurate with the cost. Objective $i i i$, to test predictions against observation, has not yet been achieved. A leak-test cell has been designed, however, and the major components for it have been fabricated.

The foregoing paragraph is a summary if progress toward achieving the original objectives of this project. One may summarize the overall accomplishments of this project as: (i) Two papers accepted for publication, one of them now in print; (ii) a Master's thesis completed by the graduate research assistant (WENDY S. SMITH); (iii) Two oral presentations made to cognizant personnel at NASA/KSC (on February 25, 1992 and December 1, 1992); (iv.) Four oral presentations made at scientific conferences*; (v.) The engineering design of a leak-test cell and the procurement of its major components.

The principal investigator now believes that he was unrealistic in September 1989 when he thought that the original objective $i i i$ could be achieved in the nineteen and three-quarters man-weeks of time allocated to him during the proposed project period. In retrospect, the entire grant period could well have been devoted to the design and fabrication of the test apparatus (ten man-weeks) and the taking of data from it (another ten weeks). In the mean time, the development of some analytical models seems to be necessary if only to indicate what data to measure and what values of measured data would or would not be in accord with theoretical predictions. The commitment of nine and three-quarters man-weeks (i.e. one academic year at a quarter-time rate) to the development of theory hardly seems excessive for this purpose.

*These presentations were at: the First World Congress of Nonlinear Analysts, Tampa, August 1992 (talk by JMR); the 45th Annual Meeting of the Division of Fluid Dynamics of the American Physical Society, Tallahassee, November 23, 1992 (separate talks by JMR and WSS); and the 31st Aerospace Sciences Meeting of the AIAA, Reno, Nevada, January 13, 1993 (talk by JMR). 


\subsection{OUTLINE OF THE REMAINDER OF THIS REPORT}

Section 2 of this report discusses analytical tools with which one may address the italicized question in section 1.1 above. Section 3 discusses the design and fabrication of a leak-test cell for testing the predictions against measurements. Sections 4,5 , and $\mathbf{6}$, discuss, respectively, options for follow-on work, recommendations resulting from the present work, and conclusions.

\section{MODELS AND SOME PREDICTIONS MADE BY THEM}

\subsection{INERTIA-DOMINATED FLOWS}

If a leak channel has the form of a contraction leading to a throat whose length is not long compared to its width, then one may expect that the dominant balance of forces in the fluid is between inertial reactions to streamwise accelerations and streamwise gradients of pressure. In this context, frictional effects play a secondary role and one is led to consider a frictionless model of the flow. The following two subsections discuss the predictions of the mass flow rate through a leak channel (modeled here as a simple stream filament) based on the frictionless models of the flow in the cases of liquid flow and gas flow, respectively.

2.1.1 LIQUIDS. The following subsection follows closely that of Reference 1, §II.1. Consider the steady flow of an incompressible, uniform-density, inviscid fluid through a stream filament. Then,

$$
p_{e}+\frac{\rho}{2} q^{2}=\left(p_{e}\right)_{\text {res }}
$$

in which: $p_{e} \equiv p-p_{h}$ is the difference between the pressure $p$ at an arbitrary station along the stream tube and the hydrostatic pressure $p_{h}$ that would exist at the same elevation if the fluid were at rest; $\rho$ is the mass density of the fluid; $q$ is the local stream speed; and $\left(p_{e}\right)_{\text {res }}$ is the value of $p_{e}$ in the supply reservoir (where the corresponding kinetic energy term $\rho q_{\text {res }}^{2} / 2$ is negligible). If $A$ is the local cross sectional area of the filament, then

$$
\dot{m}=\rho q A,
$$

in which $\dot{m}$ is the rate of transport of mass through the filament. If one solves (2.1) for $q$ and substitutes the result into (2.2), one obtains

$$
\dot{m}=\sqrt{2 \rho\left[\left(p_{e}\right)_{\text {res }}-p_{e}\right]} A .
$$

Equation (2.1) is due to EULER but is more frequently associated with the name of DANIEL BERNoulLI. It is natural, therefore, to refer to equation (2.3) as the BERNOULLI-EuLER prediction of the mass flow rate of a liquid through a leak channel.

2.1.2 GASES. The following subsection is an abridgement of $\S I I I .1$ of Reference 1. If one neglects gravitational effects, the appropriate generalization of the BERNOULLI-EULER equation for steady compressible flow is

$$
\frac{p}{\rho}+e+\frac{q^{2}}{2}=\frac{p_{0}}{\rho_{0}}+e_{0}
$$

in which the subscript " 0 " denotes evaluation of the indicated quantity at an upstream reservoir (where, as before, the fluid is at rest) and $e$ is the specific thermometric internal energy of the gas. If one assumes that the fluid is thermally and calorically perfect and that the thermodynamic processes that take place along the stream tube are isentropic, one may show, after seven lines of algebra ( $c f$. Reference $1, \S$ III.1) that

$$
\dot{m}=\sqrt{\frac{2}{\gamma-1}} a_{0} \rho_{0} \sqrt{\left(\frac{p}{p_{0}}\right)^{2 / \gamma}-\left(\frac{p}{p_{0}}\right)^{(\gamma+1) / \gamma}},
$$

in which $a_{0}$ is the local sound speed in the reservoir and $\gamma$ is the ratio $c_{p} / c_{v}$ of the specific heat at constant pressure $c_{p}$ to the specific heat at constant volume $c_{v}$. Equation (2.5) is equivalent to an equation in section 5 
of an 1839 paper by A.J.C.B. DE SAint-VEnant \& , L. WANTZEL (Reference 6). If one regards the right member of (2.5) as a function of the single variable $p / p_{0}$ and maximizes it by means of the usual method of differential calculus (i.e. setting the first derivative equal to zero), one finds that there is a maximum at the value

$$
\frac{p}{p_{o}}=\left(\frac{2}{\gamma+1}\right)^{\gamma /(\gamma-1)}
$$

(an equation that appears on page 94 of Reference 6). The corresponding maximum value of $\dot{m}$ is

$$
(\dot{m})_{\max }=\rho_{0} a_{0} A\left(\frac{2}{\gamma+1}\right)^{(1 / 2)(\gamma+1) /(\gamma-1)} .
$$

One may express $a_{0}$, the speed of sound in the rervoir, in terms of $T_{0}$, the absolute temperature there, and the specific heats by means of the standard formula

$$
a_{0}^{2}=\gamma\left(c_{p}-c_{v}\right) T_{0}
$$

In view of $(2.8)$, one may write $(2.7)$ in the equivalent form

$$
(\dot{m})_{\max }=p_{0} A \sqrt{\frac{\gamma}{\left(c_{p}-c_{v}\right) T_{0}}}\left(\frac{2}{\gamma+1}\right)^{(1 / 2)(\gamma+1) /(\gamma-1)} .
$$

The observation that $\dot{m}$ is maximized by the value given in (2.6) for the pressure was given a physicalinterpretation by OSBORNE REYNOLDS (Reference 7). REYNOLDS noted that the pressure given by (2.6) corresponds to a station where the MACH number equals one. Downstream of this point, the flow is supersonic and signals arising from downstream (such as those due to reductions in the remote receiver pressure) can not propagate upstream. Many authors refer to flow in this state as choked.

2.1.2 LIQUID TO GAS MASS FLOW RATE RATIO VERSUS RESERVOIR PRESSURE. If one divides the expression (2.3) for $\dot{m}$ for liquids by the expression (2.9) for $(\dot{m})_{\max }$ for gases and cancels the common factor $A$ in the numerator and denominator, one obtains, after simplification,

$$
\frac{(\dot{m})_{l}}{(\dot{m})_{g}}=\sqrt{\frac{\left[\left(p_{e}\right)_{\text {res }}-p_{e}\right]_{l}}{p_{0 g}} \frac{\left(c_{p}-c_{v}\right) T_{0 g} \rho_{l}}{p_{0 g}} \frac{2}{\gamma}\left(\frac{\gamma+1}{2}\right)^{(\gamma+1) /(\gamma-1)}},
$$

in which the subscripts $l$ and $g$ stand for "liquid" and "gas", respectively, and choked flow for the gas is assumed. Equation (2.10) takes a particularly simple form when one restricts attention to the case

$$
\frac{\left[\left(p_{e}\right)_{\mathrm{res}}-p_{e}\right]}{p_{0 g}}=1
$$

Equation (2.11) asserts that the difference between the inlet and the outlet pressures in the liquid is the same as the reservoir pressure in the gas. The numerator in the left member of (2.11) plays the role of driving pressure in a liquid flow and the denominator plays the role of the driving pressure in the choked flow of a gas*. By equating these two driving pressures, one can compare mass flow rates under similar conditions. Under these assumptions, equation (2.10) becomes

$$
\frac{(\dot{m})_{l}}{(\dot{m})_{g}}=\sqrt{\frac{\left(c_{p}-c_{v}\right) T_{0 g} \rho_{l}}{p_{0 g}} \frac{2}{\gamma}\left(\frac{\gamma+1}{2}\right)^{(\gamma+1) /(\gamma-1)}},
$$

*In the case of choked flow of a gas, a drop in the remote receiver pressure does not affect the flow at the throat since the flow between the throat and the remote receiver is supersonic. It follows that a drop in the remote receiver pressure below the threshhold of choking does not result in any further increase in mass flow rate. In this sense, the difference between the reservoir and the remote receiver pressures is not to be interpreted as the driving pressure for the choked flow of a gas. 
A plot of ratio $(\dot{m})_{l} /(\dot{m})_{g}$ predicted by $(2.10)$ is given in Figure 1 below.

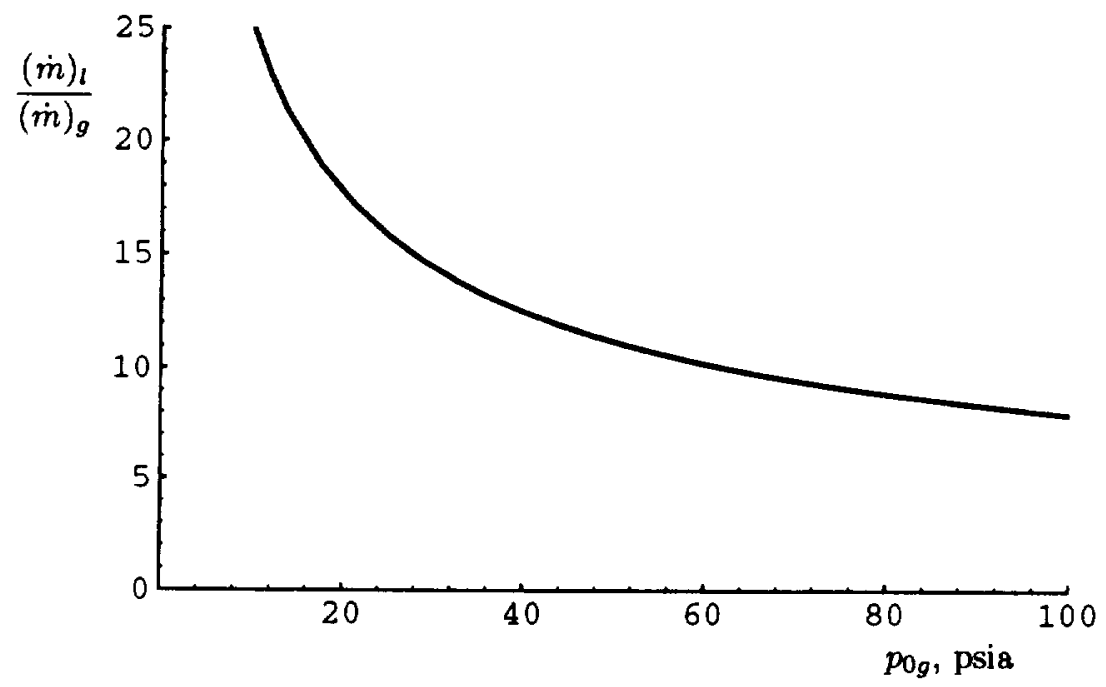

Figure 1. Ratio of mass flow rates according to (2.10). The gas that flows through the channel is assumed to be helium at the temperature of liquid nitrogen at its normal boiling point (namely $77.34^{\circ} \mathrm{K}$ ). Thus, the gas constant is taken to be $c_{p}-c_{v}=2077.69 \mathrm{~J} /\left(\mathrm{kg} \cdot{ }^{\circ} \mathrm{K}\right)$ and the ratio specific heats is taken to be $\gamma=5 / 3$. The liquid that flows through the same channel is assumed to be liquid hydrogen. Its density is thus taken to be $\rho=70.780 \mathrm{~kg} / \mathrm{m}^{3}$.

According to the equation of state of an ideal gas,

$$
\frac{\left(c_{p}-c_{v}\right) T}{p}=\frac{1}{\rho}
$$

It follows that the factor $\left(c_{p}-c_{v}\right) T_{0 g} \rho_{l} / p_{0 g}$ under the radical in $(2.10)$ is equal to $\rho_{l} / \rho_{g}$, a quotient that is large compared to one. In the meantime, the other factors under the radical in (2.10) are of the order of one. The observation that the ordinates in the Figure 1 are large compared to one is, therefore, consistent with one's expectations.

\subsection{FRICTION-DOMINATED FLOWS}

2.2.1 LIQUIDS. In the event that a leak channel is the region between a scratch in a plastic gasket and a polished metal plate against which it is pressed, one is led to consider fully developed laminar flow of a liquid through a pipe of noncircular cross section, particularly one in the form of a flat bottomed crescent. The following paragraph is a reproduction of §1I.2.1 of Reference 1.

Consider a steady flow with parallel streamlines. Let $(x, y, z)$ be cartesian axes oriented so that the $z$-axis is in the streamwise direction. If $(u, v, w)$ are the corresponding components of the velocity vector, then $u=v=0$ and $w=w(x, y)$. Under these conditions, the three components of the NAVIER-STOKES equations reduce to

$$
\frac{\partial p_{e}}{\partial x}=\frac{\partial p_{e}}{\partial y}=0 \quad, \quad 0=-\frac{\partial p_{e}}{\partial z}+\mu\left(\frac{\partial^{2} w}{\partial x^{2}}+\frac{\partial^{2} w}{\partial y^{2}}\right),
$$

in which $p_{e}$ is the excess pressure, as defined earlier. The first two equations imply that $p_{e}$ depends (at worst) on the single variable $z$, and so one may write the third equation in the equivalent form

$$
\frac{1}{\mu} \frac{d p_{e}}{d z} \equiv K=\frac{\partial^{2} w}{\partial x^{2}}+\frac{\partial^{2} w}{\partial y^{2}} \equiv \nabla^{2} w
$$


Now the leftmost member of (2.13) is independent of $x$ and $y$ and the rightmost member is independent of $z$. It follows that $K$ is a constant. Let the boundary contour be denoted by the symbol $\mathcal{C}$. Then the no-slip boundary condition asserts that

$$
w=0 \text { on } \mathcal{C} \text {. }
$$

The system consisting of the field equation (2.13) and the boundary condition (2.14) defines the boundaryvalue problem for the fully developed laminar flow of a liquid through a pipe of arbitrary cross section.

2.1.1.1 Cross section in the form of a hyperbolic crescent. Let $H$ and $h$ be given lengths subject to the condition

$$
0 \leq h \leq H .
$$

The locus of points in the $x-y$ plane that satisfy the equation

$$
\frac{y^{2}}{h^{2}}-\frac{x^{2}}{(h / \sqrt{3})^{2}}=1
$$

is a hyperbola whose asymptotes are straight lines passing through the origin with slopes $\pm \sqrt{3}$. The region $\mathcal{S}$ between the hyperbola described by (2.16) and the horizontal line described by $y=-H$ is a flat-bottomed hyperbolic crescent and is described by the inequalities

$$
\begin{array}{cl}
-H & \leq y \leq-\sqrt{3 x^{2}+h^{2}} \\
-\sqrt{\left(H^{2}-h^{2}\right) / 3} & \leq x \leq \sqrt{\left(H^{2}-h^{2}\right) / 3}
\end{array}
$$

A velocity distribution $w=w(x, y)$, which satisfies the differential equation (2.13) and the boundary condition (2.14) (the coutour $\mathcal{C}$ being the boundary of the flat-bottomed crescent $\mathcal{S}$ ) is given by the formula

$$
w=-K(y+H)\left(y^{2}-3 x^{2}-h^{2}\right) /(4 H) .
$$

One can tell by inspection of (2.19) that $w$ vanishes on the line $y=-H$. On the hyperbola defined by (2.16), moreover, $y^{2}=3 x^{2}+h^{2}$. From this observation, one may verify that $w$ vanishes on the hyperbola. These two observations confirm that $w$ satisfy the boundary condition (2.14). Finally, the application of the operator

$$
\frac{\partial^{2}}{\partial x^{2}}+\frac{\partial^{2}}{\partial y^{2}}
$$

to the right member of (2.19) takes that expression to $K$. In this way, one may verify that $(2.19)$ is an exact solution of the boundary-value problem defined by (2.13) and (2.14) for this geometry.

The mass flow rate $\dot{m}$ through the hyperbolic crescent $\mathcal{S}$ may be calculated by evaluating the integral

$$
\dot{m}=\iint_{S} \rho v d x d y .
$$

One may evaluate the integral (2.20) analytically and the result, after a laborious calculation, is (Reference 1, equation (25))

$$
\dot{m}=\frac{-\rho K H^{4}}{2 \sqrt{3}}\left\{\sqrt{1-\left(\frac{h}{H}\right)^{2}}\left[\frac{1}{30}-\frac{3}{20}\left(\frac{h}{H}\right)^{2}-\frac{2}{15}\left(\frac{h}{H}\right)^{4}\right]+\frac{1}{4}\left(\frac{h}{H}\right)^{4} \cosh ^{-1}\left(\frac{H}{h}\right)\right\} .
$$

The cross sectional area $A$ of the crescent $\mathcal{S}$ equals the value of the integral

$$
A=\iint_{S} d x d y
$$


The integral (2.22) may also be evaluated analytically and the result, after a slightly less laborious calculation, is (Reference 1 , equation (24))

$$
A=\frac{H^{2}}{\sqrt{3}}\left[\sqrt{1-\left(\frac{h}{H}\right)^{2}}-\left(\frac{h}{H}\right)^{2} \cosh ^{-1}\left(\frac{H}{h}\right)\right] .
$$

In applications if this model to the flow of liquid through a leak channel formed by a scratch in a seal, one may wish to express the parameters $h$ and $H$ in terms of other parameters, such as the corner angle $\tau$ of the crescent and the base width $2 a$. The following formulas relate $\tau$ and $a$ to $h$ and $H$ :

$$
H=\frac{3 a}{\tan (\tau)} \quad, \quad h=\frac{3 a}{\tan (\tau)} \sqrt{1-\frac{1}{3} \tan ^{2}(\tau)}
$$

2.2.1.2 Formulas and tables for application of the hyperbolic-crescent model. In applications of the above formulas, there may be circumstances in which one may wish to express the mass flow rate $\dot{m}$, the cross sectional area $A$, the maximum streamwise velocity $w_{\max }$, and a suitable flow REYNOLDS number $R e$ in terms of $a$ (the half width of the base of the crescent or, alternatively, the half width of the scratch it represents), $\tau$ (the corner angle of the crescent), $-d p_{e} / d z$ (the pressure drop per unit distance in the direction of the flow), and fluid properties such as the viscosity $\mu$ and mass density $\rho$. The following subsection provides formulas and tables which enable one to carry out such calculations.

I will begin with the formula for the mass flow rate. If one eliminates $H$ and $h$ from (2.21) by means of $(2.24 a, b)$ and restores the original meaning of $K$ as defined by (2.13), one obtains, after reductions,

$$
\dot{m}=\frac{1}{\nu}\left(-\frac{d p_{e}}{d z}\right) a^{4} f(\tau),
$$

in which $\nu \equiv \mu / \rho$ is the kinematic viscosity and the nondimensional function $f(\tau)$ is defined by

$$
\begin{aligned}
f(\tau) \equiv & \frac{1}{2 \sqrt{3}}\left(\frac{3}{\tan (\tau)}\right)^{4}\left\{\frac { \operatorname { t a n } ( \tau ) } { \sqrt { 3 } } \left[\frac{1}{30}-\frac{3}{20}\left(1-\frac{\tan ^{2}(\tau)}{3}\right)\right.\right. \\
& \left.\left.-\frac{2}{15}\left(1-\frac{\tan ^{2}(\tau)}{3}\right)^{2}\right]+\frac{1}{4}\left(1-\frac{\tan ^{2}(\tau)}{3}\right)^{2} \cosh ^{-1}\left[\left(1-\frac{\tan ^{2}(\tau)}{3}\right)^{-1 / 2}\right]\right\} .
\end{aligned}
$$

Table 1 furnishes numerical values of the auxiliary function $f(\tau)$ for values of the corner angle $\tau$ in the range $0 \leq \tau \leq 60^{\circ}$.

\begin{tabular}{|c|l|l|l|l|}
\hline \multicolumn{5}{|c|}{ Table 1. Auxiliary functions for the calculation of $\dot{m}, A, w_{\max }$, and $R e$} \\
\hline$\tau$, degrees & \multicolumn{1}{|c|}{$f(\tau)$} & \multicolumn{1}{|c|}{$g(\tau)$} & \multicolumn{1}{|c|}{$F(\tau)$} & \multicolumn{1}{|c|}{$G(\tau)$} \\
\hline $\mathbf{0}$ & $\mathbf{0 .}$ & 0. & 0. & 0. \\
5 & 0.00000638316 & 0.0583556 & 0.000239272 & 0.0000104735 \\
10 & 0.0000523928 & 0.117796 & 0.000972862 & 0.0000859943 \\
15 & 0.000184696 & 0.179497 & 0.00225041 & 0.000303323 \\
20 & 0.000466105 & 0.244831 & 0.00416296 & 0.000766151 \\
25 & 0.0009898 & 0.315524 & 0.00685794 & 0.00162903 \\
30 & 0.00190439 & 0.393888 & 0.0105661 & 0.00313955 \\
35 & 0.00346221 & 0.483240 & 0.0156499 & 0.00572336 \\
40 & 0.00612073 & 0.588705 & 0.022695 & 0.0101575 \\
45 & 0.0107785 & 0.718962 & 0.0326921 & 0.0179973 \\
50 & 0.0194089 & 0.890832 & 0.0474388 & 0.0327617 \\
55 & 0.0372192 & 1.14572 & 0.0705729 & 0.0643687 \\
60 & 0.0866025 & 1.73205 & 0.111111 & 0.19245 \\
\hline
\end{tabular}


The tabulated value of $f(0)$ represents a limit as $\tau \rightarrow 0$ and the same remark applies to the tabluated values of all of the other auxiliary functions listed in Table 1.

The next formula to be derived is the one for the cross sectional area $A$. If one eliminates $H$ and $h$ from (2.23) by means of $(2.24 a, b)$, one obtains, after reductions,

$$
A=a^{2} g(\tau),
$$

in which the nondimensional function $g(\tau)$ is defined by

$$
g(\tau) \equiv \frac{1}{\sqrt{3}}\left(\frac{3}{\tan (\tau)}\right)^{2}\left\{\frac{\tan (\tau)}{\sqrt{3}}-\left(1-\frac{\tan ^{2}(\tau)}{3}\right) \cosh ^{-1}\left[\left(1-\frac{\tan ^{2}(\tau)}{3}\right)^{-1 / 2}\right]\right\}
$$

Table 1 furnishes numerical values of the auxiliary function $g(\tau)$ for values of the corner angle $\tau$ in the range $0 \leq \tau \leq 60^{\circ}$. As was the case with the auxiliary function $f(\tau)$, the tabluated value of $g(0)$ represents a limit as $\tau \rightarrow 0$.

The next formula to be derived is the one for the maximum value $w_{\max }$ of the streamwise velocity $w$. Inspection of (2.19) shows that for any $y$-elevation in the crescent, the maximum velocity at that elevation will occur at $x=0$. If one sets $x=0$ in (2.19) and maximized it by the usual method of differential calculus (differentiating it with respect to $y$ and setting the result equal to zero), one finds that there is a maximum at the elevation

$$
y_{\operatorname{maxw}}=-\left(H+\sqrt{H^{2}+3 h^{2}}\right) / 3 .
$$

If one substitutes $(x, y)=\left(0, y_{\max w}\right)$ into $(2.19)$, one obtains, after rearrangement,

$$
w_{\max }=-\frac{K H^{2}}{4}\left\{1-(1 / 3)\left[1+\sqrt{1+3(h / H)^{2}}\right]\right\}\left\{\left(1 / 3^{2}\right)\left[1+\sqrt{1+3(h / H)^{2}}\right]^{2}-(h / H)^{2}\right\} .
$$

If one eliminates $H$ and $h$ from (2.30) by means of $(2.24 a, b)$, and restores the original meaning of $K$ defined by (2.13) one obtains, after reductions,

$$
w_{\max }=\frac{a^{2}}{\mu}\left(-\frac{d p_{e}}{d z}\right) F(\tau)
$$

in which the nondimensional function $F(\tau)$ is defined by

$$
F(\tau) \equiv \frac{1}{4 \tan ^{2}(\tau)}\left[2-\sqrt{4-\tan ^{2}(\tau)}\right]\left\{\frac{1}{3}\left[2-\sqrt{4-\tan ^{2}(\tau)}\right]^{2}-\left[3-\tan ^{2}(\tau)\right]\right\} .
$$

Table 1 furnishes numerical values of the auxiliary function $F(\tau)$ for values of the corner angle $\tau$ in the range $0 \leq \tau \leq 60^{\circ}$. As was the case with the auxiliary function $f(\tau)$, the tabluated value of $F(0)$ represents a limit as $\tau \rightarrow 0$.

The next formula to be derived is the one for a suitable REYNolds number of the flow. The maximum thickness of the crescent (measured normal to its flat face) equals $H-h$. If one takes this length and the velocity $w_{\max }$ in the definition of the REYNOLDS number, one has

$$
R e=\frac{\rho w_{\max }(H-h)}{\mu}=\frac{\left.\rho w_{\max } H[1-(h / H)]\right)}{\mu} .
$$

If one eliminates $w_{\max }, H$, and $h$, from this equation by means of $(2.31),(2.24 a)$, and (2.24b), respectively, one obtains, after reductions

$$
R e=\frac{\rho a^{3}}{\mu^{2}}\left(-\frac{d p_{e}}{d z}\right) G(\tau),
$$


in which the nondimensional function $G(\tau)$ is defined by

$$
G(\tau) \equiv F(\tau)\left(\frac{3}{\tan (\tau)}\right)\left(1-\sqrt{1-\frac{\tan ^{2}(\tau)}{3}}\right)
$$

Table 1 furnishes numerical values of the auxiliary function $G(\tau)$ for values of the corner angle $\tau$ in the range $0 \leq \tau \leq 60^{\circ}$. As was the case with the auxiliary function $f(\tau)$, the tabulated value of $G(0)$ represents a limit as $\tau \rightarrow 0$.

2.2.1.3 Calculation examples. Some calculation examples may help to illustrate the application of the foregoing formulas and tables. Suppose that the rate of drop of pressure in a leak channel has the value

$$
-\frac{d p_{e}}{d z}=1 \frac{\mathrm{atm}}{\mathrm{mm}}=1.01325 \times 10^{8} \frac{\mathrm{N}}{\mathrm{m}^{3}}
$$

Suppose further that the leak rate is measured at a remote receiver where the temperature and the pressure have values that correspond to the U.S. standard atmosphere at sea level, i.e. $T_{\mathrm{std}}=15^{\circ} \mathrm{C}=288.15^{\circ} \mathrm{K}$ and $p_{\text {std }}=1 \mathrm{~atm}=1.01325 \times 10^{5} \mathrm{~N} /(\mathrm{m})^{2}$. From the law of conservation of mass applied to a stream tube, we have

$$
(\dot{m})_{\text {leak channel }}=(\dot{m})_{\text {remote receiver }}=\rho_{\text {std }} \dot{V}_{\text {std }}
$$

It follows from the equation of state of an ideal gas that

$$
\rho_{\text {std }}=\frac{p_{\text {std }}}{\left(c_{p}-c_{v}\right) T_{\text {std }}}=\frac{p_{\text {std }}}{(\mathcal{R} / M) T_{\text {std }}}
$$

in which $\mathcal{R}=8,317 . \mathrm{Nm} /\left(\mathrm{kg}-\right.$ mole $\left.^{\circ} \mathrm{K}\right)$ is the universal gas constant and $M$ is the molecular weight of the gas. It follows from (2.37) that

$$
(\dot{m})_{\text {leak channel }}=\frac{p_{\text {std }} \dot{V}_{\text {std }}}{(\mathcal{R} / M) T_{\text {std }}}
$$

The molecular weight $M$ is fixed by the choice of working fluid. The standard temperature and pressure, moreover, are also known. The only parameter left unspecified in the right member of (2.39) is the volume flow rate $\dot{V}_{\text {std }}$ into the remote receiver, which I will specify as $\dot{V}_{\text {std }}=10(\mathrm{~cm})^{3} / \mathrm{min}=1.66667 \times 10^{-7}(\mathrm{~m})^{3} / \mathrm{s}$. An operational criterion for leak tightness of a valve meant to control the flow of liquid hydrogen might be

\begin{tabular}{|c|c|c|c|}
\hline \multicolumn{4}{|c|}{$\begin{array}{l}\text { Table 2. Properties of liquid hydrogen and liquid nitrogen } \\
\text { at their normal boiling points }\end{array}$} \\
\hline Substance & $\rho, \mathrm{kg} /(\mathrm{m})^{2}$ & $\mu, \mathrm{N} \mathrm{s} /(\mathrm{m})^{2}$ & $M, \mathrm{~kg} /(\mathrm{kg}$ mole $)$ \\
\hline Hydrogen & 70.78 & $1.320 \times 10^{-5}$ & 2.01594 \\
\hline Nitrogen & 808.8 & $16.4 \times 10^{-5}$ & 28.0134 \\
\hline
\end{tabular}
ten standard cubic centimeters per minute and the choice of parameters in the present calculation examples applies to this case.

The fluid properties assumed in the present calculation examples were taken from References 8 and 9 and are summarized in Table 2

To use the equations for $\dot{m}, A, w_{\max }$, and $R e$ in the hyperbolic crescent model, one must specify an effective value of the corner angle. In the present calculations, I have assumed the value

$$
\tau=30^{\circ}
$$


Equation (2.25) may be written in the equivalent form

$$
a=\left(\frac{\dot{m} \mu}{\left(-d p_{e} / d z\right) f(\tau)}\right)^{1 / 4} .
$$

Table 3 gives the results of the sample calculations

Table 3. Calculated values of the channel half span, cross-sectional area, maximum flow speed, and REYNOLDS number

\begin{tabular}{|c|c|c|c|c|}
\hline Substance & $a, \mu \mathrm{m}$ & $A,(\mu \mathrm{m})^{2}$ & $w_{\max }, \mathrm{m} / \mathrm{s}$ & $R e$ \\
\hline Hydrogen & 10.82 & 46.15 & 9.495 & 163.7 \\
Nitrogen & 12.00 & 56.73 & 0.9402 & 16.53 \\
\hline
\end{tabular}

The order of magnitude of the calculated REYNOLDS numbers are consistent with the assumption of laminar flow.

2.2.1.4 Cross section in the form of a circular crescent. The Master's thesis of WENDY S. SMITH (whose work was supported by the present grant in the form of a research assistantship during the period September 1991 to September 1992) it titled "Fully-developed laminar flow through a pipe whose cross section is a flat bottomed circular crescent" (Reference 3). This work presents an exact solution of boundary-value problem described by equations (2.13) and (2.14) above in the case when the boundary contour $\mathcal{C}$ is described by the inequalities

$$
\begin{gathered}
0 \leq y \leq y_{\mathrm{arc}}(x) \\
-a \leq x
\end{gathered}
$$

in which $y_{\operatorname{arc}}(x)$ satisfies the equation of a circle

$$
x^{2}+\left(y_{\mathrm{arc}}(x)-y_{0}\right)^{2}=R^{2}
$$

whose center $y_{0}$ is situated at

$$
y_{0}=-a \cot (\tau)
$$

and whose radius $R$ is determined by the equation

$$
a=R \sin (\tau) .
$$

The change of variables $(x, y) \rightarrow(\xi, \eta)$ defined by the equations

$$
\xi \equiv \tanh ^{-1}\left(\frac{2 a x}{a^{2}+x^{2}+y^{2}}\right) \quad, \quad \eta \equiv \tan ^{-1}\left(\frac{2 a y}{a^{2}-x^{2}-y^{2}}\right)
$$

or, equivalently, by

$$
x=\frac{a \sinh \xi}{\cosh \xi+\cos \eta} \quad, \quad y=\frac{a \sin \eta}{\cosh \xi+\cos \eta}
$$

is convenient. SMITH shows that the geometric figure in the plane of the variables $(\xi, \eta)$ that corresponds to the crescent in the plane of the variables $(x, y)$ is an infinite strip parallel to the $\xi$-axis. Specifically, the image of the region defined by the inequalties $(2.42),(2.43)$ is the region

$$
\begin{gathered}
-\infty<\xi<\infty \\
0<\eta<\tau .
\end{gathered}
$$

The system of equations $(2.47 a, b)$ is convenient for calculating $(\xi, \eta)$ from given values of $(x, y)$ and the system $(2.48 a, b)$ is convenient for calculating $(x, y)$ from given values of $(\xi, \eta)$. The system of variables $(\xi, \eta)$ is thus interchangable with the system $(x, y)$ as a representation of position in the flow. 
SMITH's formulas for the streamwise velocity distribution at an arbitrary position on the channel cross section are as follows

$$
\begin{aligned}
w(\xi, \eta)= & \frac{K y^{2}}{2}-\frac{K a^{2}}{4 \tau} \int_{\xi=-\infty}^{\infty}\left(\frac{\sin \tau}{\cosh X \cos \tau}\right)^{2} \frac{\sin (\pi \eta / \tau)}{\cosh [(\pi / \tau)(X-\xi)]+\cos (\pi \eta / \tau)} d X \\
= & \frac{K}{4}\left[x^{2}+(y+a \cot \tau)^{2}-a^{2} \csc ^{2} \tau\right] \\
& +\frac{K a^{2}}{8 \tau} \int_{\xi=-\infty}^{\infty} \frac{1}{\cosh ^{2}(X / 2)} \frac{\sin (\pi \eta / \tau)}{\cosh [(\pi / \tau)(X-\xi)]-\cos (\pi \eta / \tau)} d X
\end{aligned}
$$

The right members of (2.51) and (2.52) are both valid representations of $w(\xi, \eta)$ and their regions of validity overlap, but they are convenient in different regions of the flow. Thus, the right member of line (2.51) is convenient for the calculation of $w(\xi, \eta)$ in the region $0 \leq \eta \leq \tau / 2$ and the right member of line (2.52) is convenient for the calculation of $w(\xi, \eta)$ in the region $\tau / 2 \leq \eta \leq \tau$.

SMITH'S thesis furnishes contours of constant value of $w(x, y)$ for various corner angles in the range $0 \leq$ $\tau \leq 90^{\circ}$. As one might expect, disagreement between the solution for $w(x, y)$ in the case of the flat-bottom circular crescent and the solution for $w(x, y)$ in the case of the flat-bottom hyperbolic crescent vanishes in the limit $\tau \rightarrow 0$. This agreeement verifies the absence of gross algebraic errors.

The reader is referred to Reference 3 for further results derived from the system (2.51) and (2.52). Such additional results include calculation of the mass flow rates by numerical integration and calculation of the circumferentially averaged skin friction coefficient as a function of the pipe-flow REYNOLDS number.

2.2.1 GASES. Reference 2, which was written during the present grant peroid, is titled "The present status of the theory of the flow of a compressible viscous fluid through a pipe or parallel-wall channel." According to its abstract

In pipe-flow geometry, the differential equations of motion of a viscous gas do not lend themselves to analytic solution even when they are subject to the boundary-layer approximation. The difficulty stems from the lack of a main-stream region that abuts the region where frictional effects are important and where the [streamwise] pressure distribution is known. Thus, most classical models of gas flow through a pipe are based on the assumptions that the cross-stream integrals of various products of the flow variables may always be replaced by algebraic expressions involving the products of their cross-stream averages and that the relationship between the skin friciton coefficient and REYNOLDS number does not depend significangly upon the compressibility of the fluid. The present work is a derivation of such a one-dimensional model which aims to avoid unnecessary assumptions and to enumerate the unavoidable ones with some precision.

This paper was presented at the First World Congress of Nonlinear Analysts held in Tampa, August 1992, and the written version of the paper has been accepted for publication in the proceedings. The reader is referred to that paper for details of the derivation and interpretation of the classical one-dimensional model of compressible flow through a pipe and for references to the earlier literature one the subject.

As in the case of frictioness flow through a convergent-divergent nozzle, there are two regimes of the flow, fully subsonic flow and choked flow (flow with sonic conditions at the outlet). The choked flow case is the generic one and is the one whose equations I will present here. According to the model presented in Reference 2 , the mass flow rate $\dot{m}$ through the channel obeys an equation of the form

$$
\dot{m}=t_{\text {in }}^{1 /(\gamma-1)} \overline{p_{0}} \frac{\gamma}{\gamma-1} \sqrt{\frac{2}{c_{p} \overline{T_{0}}}\left(1-t_{\text {in }}\right)} A
$$


The overbar denotes the cross-stream average of the indicated quantity. The subscript zero denotes the value of the indicated quantity in the supply reservoir. The flow between the supply reservoir (where the kinetic energy term is negligible) and the inlet to the leak channel (where it is not) is presumed to be isentropic. The subscript "in" denotes evaluation of the indicated quantity at the inlet to the leak channel. The symbol $t$ is a shorthand for the ratio $\bar{T} / \overline{T_{0}}$. All of the other symbols are used in a manner consistent with earlier uses of the same symbol in this resport. Manipulation of the equations of mass, momentum, and energy leads, at length, to the transcendental equation

$$
\begin{array}{r}
\frac{1}{4 \gamma \mu\left(\overline{T_{0}}\right)}\left[(\gamma+1)(-) \beta\left(t_{\mathrm{in}}, 2 /(\gamma+1), 1-n, 0\right)+(\gamma-1) \beta\left(t_{\mathrm{in}}, 2 /(\gamma+1), 1-n,-1\right)\right] \\
=\frac{L}{\dot{m}} \iint_{S} \operatorname{div}[\operatorname{grad}(w / \bar{w})] d A
\end{array}
$$

in which: $L$ is the length of the leak channel; $n$ is the exponent in a power-law formula for the dependence of the viscosity upon the absolute temperature viz

$$
\mu(\bar{T}) \approx \mu_{0}\left(\bar{T} / \overline{T_{0}}\right)^{n}
$$

$\left(n \approx 0.666\right.$ and $\mu_{0}=\mu\left(\overline{T_{0}}\right) \approx 1.870 \times 10^{-5} \mathrm{~N} \mathrm{~s} / \mathrm{m}^{2}$ for helium); and $\beta\left(z_{0}, z_{1}, a, b\right)$ is the generalized beta function defined by

$$
\beta\left(z_{0}, z_{1}, a, b\right) \equiv \int_{z_{0}}^{z_{1}} t^{a-1}(1-t)^{b-1} d t
$$

For helium, $\gamma=5 / 3$. If one assumes that the cross section of the leak channel is a hyperbolic crescent and one models the double integral in the right member of (2.55) by its value in the case of liquid flow, one arrives at the formula

$$
\iint_{\mathcal{S}} \operatorname{div}[\operatorname{grad}(w / \bar{w})] d A=-\frac{[g(\tau)]^{2}}{f(\tau)} .
$$

in which $f(\tau)$ and $g(\tau)$ are tabulated in Table 1 of section 2.2.1.2.

In an application to the prediction of leak flow rates, all of the symbols in (2.54) are given data with the exception of $t_{\mathrm{in}}$. Equation (2.54) then constitutes a transcendental equation that is to be solved numerically for that one unknown. Once $t_{\text {in }}$ has been found numerically, the corresponding value of the mass flow rate $\dot{m}$ follows from equation (2.53).

For further discussion of this calculation method (including the discussion of the fully subsonic case), the reader is referred to Reference 2.

\subsection{EFFECTS NEGLECTED IN THE MODELS FORMULATED THUS FAR}

The main effects neglected in the models formulated thus far are:

1. The effects of adiabatic boiling in the flow of a liquid through a leak channel. If a liquid is already at its boiling point at the inlet to a leak channel and the pressure decreases in the direction of flow (as it must), then at some downstream station, the pressure may dip below the vapor pressure of the liquid at that temperature and boiling would commence.

2. The effects of turbulence. For the example calculations of section 2.2.1.3, the REYNOLDS number was consistent with the assumption of laminar flow. For larger leaks-those whose volume flow rates at a remote exhaust greatly exceed $10 \mathrm{~cm}^{3} / \mathrm{s}-$ one must expect to the transition to from laminar to turbulent flow to exert a strong influence on the flow rate.

3. The effects of inlet-swirl. If the flow in the supply reservoir is not at rest but is in shearing motion (a situation that would apply if the leak springs from a pipe through which fluid is being transported), 
then one may expect a bathtub vortex to form at the inlet and to exert a significant influence on the leak rate.

\section{DESIGN AND FABRICATION OF A LEAK-TEST CELL}

\subsection{REQUIREMENTS}

The leak test cell designed during the present grant period was subject to the following requirements.

1. The cell must have a supply vessel from which fluid escapes through a controlled flaw in a gasket.

2. The leaked fluid must be captured within a containment vessel.

3. In order to minimize the number of uncontrolled leaks, there should be as few gaskets as possible.

4. There must be some means for measuring the leak rate.

\subsection{FACTORS LEADING TO THE CHOICE OF THE PRESENT CONFIGURATION}

A concept of a leak test cell that is consistent with the above requirements is one in which two cups, one inside the other, are bolted to a flat plate. The chamber enclosed by the inner cup and the plate forms a supply vessel. A controlled flaw in the gasket that separates the inner cup from the plate lets the working fluid leak into the receiver chamber bounded by the inner cup, the outer cup, and the plate. If fluid lines run from the receiver chamber through the plate to laboratory monitoring devices, then one can measure the rate of leakage directly. There is a gasket between the plate and the inner cup and another between the plate and the outer cup. A design which reduces the number of gaskets to less than two does not seem to be possible.

A scenario for the use of the leak test cell is as follows: (i) the cups are bolted to the plate at room temperature; (ii) the cell is immersed in a bath of a cryogenic fluid such as liquid nitrogen; (iii) the tests are then run at cryogenic temperatures. Between stages (i) and (ii), of course, there will be thermal contraction of the whole assembly. In particular, the proportional contraction of the gaskets will an order of magnitude greater than the proportional contraction of the steel cups. To avoid the appearance of new leak paths due to this differential shrinkage during cooldown, the design must provide for the maintenance of bolt tension despite this differential shrinkage. The solution adopted in the present design is to install BELLVILLE springs (also known as disk springs) on the bolts prior to installation of the nuts. The theory of BELLVILLE springs is over a half a century old (ALMEN \& LAXSO, 1936, Reference 10). They are avaliable in a wide variety of sheet thicknesses, cone angles, inner and outer radii, and materials. They can also be stacked in parallel, in series, or in some combination of the two. The flexibility of the BELLEvilLE spring enables the design of the test cell so that enough compliance is in the system to maintain a reasonable fraction (say $90 \%$ ) of the original room temperature bolt tension after cooldown to cryogenic temperature.

As of March 1993, the cups, and the plate have been fabricated, the BELLEviLLE springs, the gaskets, the nuts, and the bolts have been bought. Various options have been considered for running the fluid lines through the plate, but the final decision on the choice of fluid-line installation has not been made. Of course, any satisfactory choice of fittings for running the fluid lines through the plate must not introduce new leak paths. As an additional constraint, the pickups for the fluid lines inside the supply and receiver chambers should play the role of source and drain in each chamber. In particular, one pickup should be flush with the floor and the other should be flush with the ceiling.

A drawing of the leak test cell is given on the following page.

\section{RECOMMENDATIONS FOR FOLLOW-ON WORK}

1. Finish the construction and the debugging of the leak test cell, including the installation of fluid lines. 

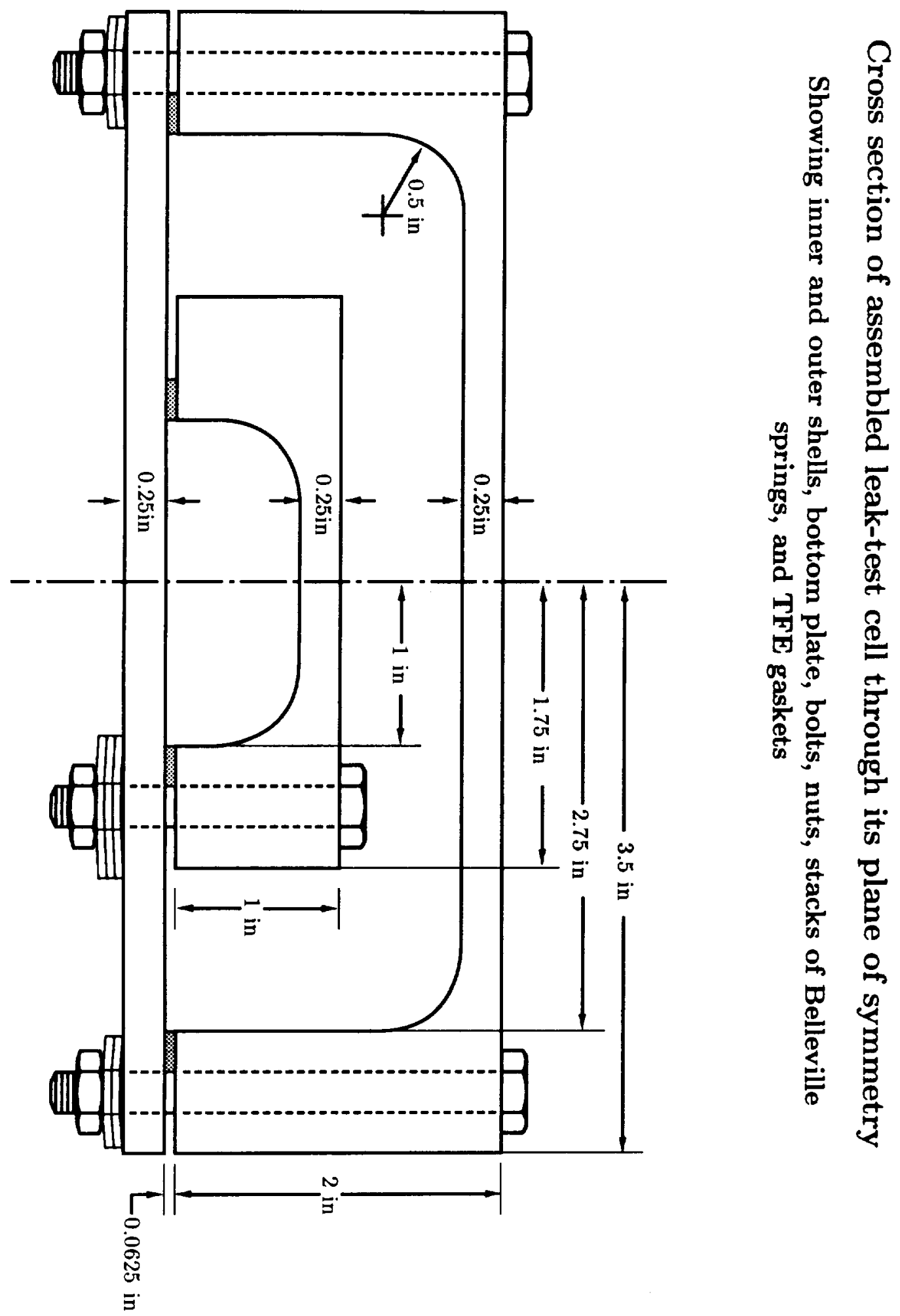
2. Attach the leak test cell to suitable instrumentation for measuring leak flow rates. At first, such instrumentation will take the form of a small flow rate measurement device. At a later time, it will probably take the form of a mass spectrometer.

3. Apply the analytical method for predicting the mass flow rate of a viscous gas to the flow of helium through a leak path at cryogenic temperature.

4. Experiment with various methods for producing a controlled flaw in a seal. As suggested by Mr. JAMES FESMIRE, such a controlled flaw make take the form of a fine wire draped across the gasket prior to final assembly of the cell. The determination of the geometry of the resulting faw is a problem that has not been solved and must be addressed before quantitative comparisons between measurements and theory
can be made.

\section{CONCLUSIONS}

1. If: (a) the flow of a gas and a liquid through a given leak path is dominated by the balance between inertia and pressure gradients; (b) the reservoir pressure for the gas is the same as the inlet-outlet pressure difference for the liquid; and (c) the gas flow is choked, then the ratio of the mass flow rate of the liquid to the mass flow rate of the gas scales with the square root of the ratio of the density of the liquid to the density of the gas in the reservoir.

2. In the case of friction dominated flow of a liquid through a uniform leak channel whose cross section is a flat bottomed hyperbolic crescent, the mass flow rate is directly proportional to the rate of drop of pressure with streamwise distance, inversely proportional to the kinematic viscosity, and proportional to the fourth power of the half width of the leak channel.

3. In the case of friction dominated flow of a liquid through a uniform leak channel whose cross section is a flat bottomed hyperbolic crescent, if the corner angle equals thirty degrees, the rate of pressure drop equals one atmosphere per milimeter, and the rate of discharge of fluid volume into a remote receiver at standard conditions is ten cubic centimeters per minute, then the half width of the leak channel is about eleven microns for hydrogen and about twelve microns for nitrogen.

\section{REFERENCES}

1. Russell, John M. 1993 Development and verification of methods for predicting flow rates through leaks in valves and couplings. AIAA Paper 93-0559, presented at the 31 Aerospace Sciences Meeting, Reno, Nevada, January 11-14, 1993.

2. RusSELL, JoHN M. 1993 The present status of the theory of the flow of a compressible viscous fluid through a pipe or parallel-wall channel. Accepted for publication in the Proceedings of the First World Congress of Nonlinear Analysts, Tampa, Florida August 19-26, 1992, Walter de Gruyter Company (to
appear).

3. SMITH, Wendy S. 1993 Fully-developed laminar flow through a pipe whose cross section is a flatbottomed circular crescent. Master's thesis submitted to the Department of Mechanical and Aerospace Engineering, Florida Institute of Technology, Winter 1993.

4. Burmeister, Lois C., Loser, John B., \& SNeegas, Eldon C. 1967 NASA contributions to advanced valve technology. NASA SP-5019 (especially chapter 2, titled "Leakage", pp 3-17, by J.W. MARR, physical chemist, General Electric).

5. SHAPIRO, ASCHER 1953 The dynamics and thermodynamics of compressible fluid flow. New York: The Roland Press (in two volumes). See especially volume 1, pp 159-189.

6. Saint-Venant, Adhémar Jean Claude Barré de \& Wantzel, Laurent 1839 Mémoires et expériences sur l'écoulement de l'air, déterminé par des différences de pressions considérables. Journal de l'ecole polytechnique (Paris), 16(27), pp 85-122.

7. ReYnolds, OsBorne 1886 On the flow of gases. Philosophical Magazine, 21, pp 155-199. [Reprinted in Papers on mathematical subjects (collected works of OsBorne REYNOLDS), 2, pp 311-320, Cambridge University Press, 1901]. 
8. MCCARTY, ROBERT D. 1975 Hydrogen technological survey-thermophysical properties. NASA SP-3089.

9. JaCobsen, R.T., Stewart, R.B., MCCARTY, R.D., \& Hanley, H.J.M. 1973 Thermophysical properties of nitrogen from the fusion line to $3500 \mathrm{R}(1944 \mathrm{~K})$ for pressures to $150,000 \mathrm{psia}\left(10342 \times 10^{5}\right.$ $\mathrm{N} / \mathrm{m}^{2}$ ). U.S. Department of Commerce, National Bureau of Standards Technical Note 648.

10. Almen, J.O. \& Lazlo, A. 1936 The uniform-section disk spring. Transactions of the ASME, 58, pp 305-314. 